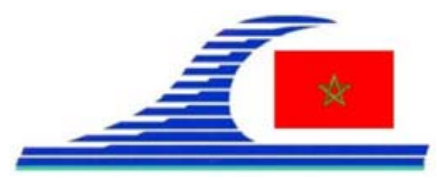

Conférence Méditerranéenne Côtière et Maritime

EDITION 2, TANGER, MAROC (2011)

Coastal and Maritime Mediterranean Conference

Disponible en ligne - http://www.paralia.fr - Available online

\title{
Impact des aménagements sur la morphodynamique du complexe lagunaire de Nador, Maroc
}

\author{
Abdelkarim LOUAYA ${ }^{1}$, Naima HAMOUMI ${ }^{2}$ \\ 1. Laboratoire Océanologie et Géodynamique des Bassins sédimentaires, Faculté des \\ Sciences, Université Mohammed V - Agdal, Rabat, Maroc. \\ abdelkarim159@yahoo.fr ; naimahamoumi@yahoo.fr
}

\section{Résumé :}

Le complexe lagunaire de Nador (littoral méditerranéen oriental), présente un intérêt particulier dans la région, aussi bien sur le plan scientifique que sur le plan socioéconomique. Le suivi de l'évolution morphodynamique de ce complexe durant la période 2005-2010, a été réalisé dans le but de connaître l’incidence des aménagements récents : fermeture de l'ancienne passe en vue de sa transformation en un port de plaisance et ouverture d'une nouvelle passe. Il fait suite et s'appuie sur les résultats de l'étude morphostructurale et géomorphologique qui a été menée dans le cadre du projet COLASU à la fois dans : le complexe lagunaire, son arrière pays et le proche plateau continental. Les résultats obtenus à l'aide de techniques variées : étude de terrain, analyse des données historiques, interprétation des photos aériennes au 1/20000 et analyse des images satellites (Landsat MSS., 1975 et 1976 ; Landsat TM ,1986, 1988, 1993 et 2010, Landsat +ETM, 2000, 2005), ont permis de confirmer le contrôle de la tectonique et du climat sur la genèse et l'évolution du complexe lagunaire et de mettre en évidence des déséquilibres sédimentaires (érosion, engraissement) au niveau de l'île barrière et de la lagune qui sont induits par les aménagements récents.

\section{Mots-clés :}

Ecosystème côtier - Géomorphologie - Morphodynamique - Impact des aménagements - Lagune de Nador- Maroc - Littoral méditerranéen

\section{Introduction}

Le complexe lagunaire de Nador situé dans le littoral méditerranéen oriental (Fig. 1), est constitué par une île barrière de $25 \mathrm{~km}$ de long dont la largeur varie de 300m dans sa partie NW à $2 \mathrm{~km}$ dans son extrémité SE et une lagune de $115 \mathrm{~km}^{2}$ de superficie dont la profondeur maximale atteint $7 \mathrm{~m}$ dans la partie centrale. Il appartient au bassin néogène subsident de Nador-Mélilla qui s'est individualisé au Miocène supérieur postérieurement à la mise en place des nappes externes rifaines.

Du fait de son intérêt scientifique et socio-économique, le complexe lagunaire a fait l'objet de nombreuses études, cependant, les rares travaux qui se sont intéressé à la géomorphologie et la morphodynamique, n’ont porté que sur l'île barrière et ses passes 
La connaissance de la Mer :

un vecteur du développement durable en Méditerranée

(ERIMISCO, 1961 ; TESSON, 1977 ; MAHJOUBI, 1991 ; IRZI \& HAMOUMI, 1997 ; IRZI et al., 1997 ; HAMOUMI \& IRZI, 1999 ; IRZI, 2002). Sachant que les différentes unités physiographiques de ce complexe sont interdépendantes et que son évolution est étroitement liée au contexte géologique et géomorphologique régional, une étude morphostructurale et géomorphologique détaillée a été menée à la fois dans: le complexe lagunaire, son arrière pays et le proche plateau continental. Entre autres résultats, cette étude a montré que durant la période 1975-2005, les activités anthropiques ont affecté de manière significative l'équilibre de ce complexe dont la genèse et l'évolution sont contrôlées par la tectonique et le climat (HAMOUMI \& LOUAYA, 2005 ; LOUAYA \& HAMOUMI, 2006 ; LOUAYA \& HAMOUMI, 2010). Il était donc intéressant de poursuivre les études afin d'appréhender l'incidence des aménagements récents : 1 ) ouverture d'une passe artificielle avec un chenal de $300 \mathrm{~m}$ de large et $6 \mathrm{~m}$ de profondeur et deux digues de calibrages de 1450 et $1350 \mathrm{~m}$ et 2) fermeture de l'ancienne passe en vue de sa transformation en un port de plaisance.

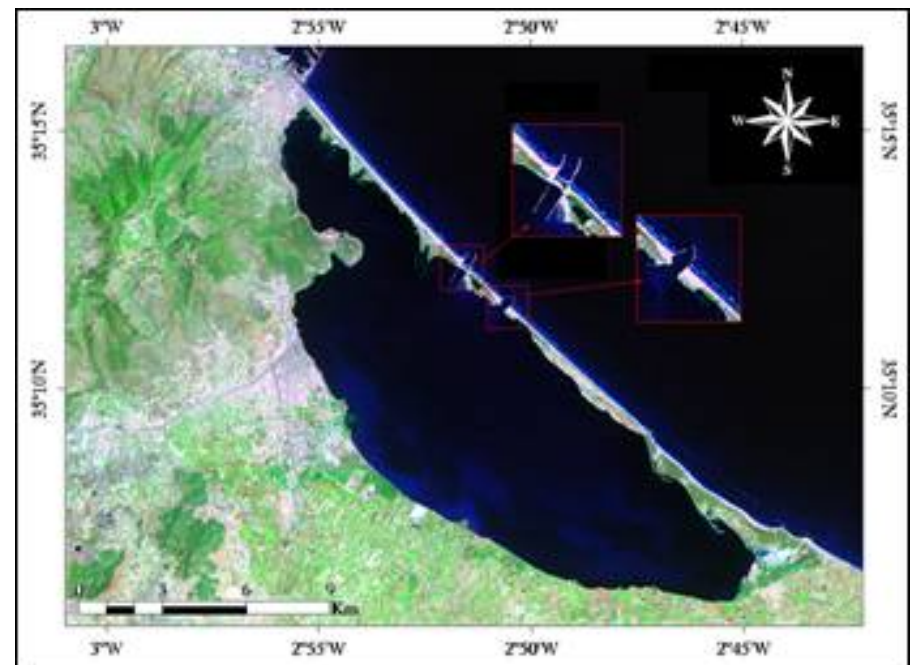

Figure 1. Situation des aménagements récents de l'île barrière de la lagune de Nador.

\section{Matériels et méthodes}

L'étude géomorphologique et morphodynamique du complexe lagunaire de Nador (littoral méditerranéen oriental Maroc) et de son arrière pays a été réalisée en intégrant des techniques variées. En plus des résultats de: l'étude de terrain, l'analyse des données historiques, l'interprétation des photos aériennes au 1/20000 et l'analyse morphostructurale (LOUAYA \& HAMOUMI, 2010), il a été procédé à l'analyse des images satellites (Landsat MSS., 1975 et 1976 ; Landsat TM ,1986, 1988, 1993 et 2010, Landsat +ETM, 2000, 2005 ), après une correction géométrique par rapport à la carte topographique $1 / 50000$ de Nador et des corrections radiométriques spatiales et spectrales adéquates à l'aide du logiciel ENVI 4.7. 


\section{Résultats}

En plus de l'actualisation des données géomorphologiques et hydrologiques des différentes unités physiographiques du complexe lagunaire pour la période 2005 - 2010, les résultats de ce travail ont permis de mettre en évidence des déséquilibres sédimentaires au niveau de l'île barrière et de la lagune qui sont induits par les aménagements récents et les activités anthropiques (Fig. 2 et 3).

- La façade marine de l'île barrière est le siège d'un engraissement important derrière la digue Ouest de la passe artificielle créée en 2009 et d'un démaigrissement au niveau de l'extrémité NW et toute la partie comprise entre la digue Est de la passe artificielle et Kariat Arekmane.

- La façade continentale de l'île barrière, montre un engraissement derrière la digue Est de la passe artificielle aménagée en 2009 et de part et d'autre du double tombolo.

- La lagune est le siège d'un comblement dans ses extrémités NW et SE.

- La bordure continentale de la lagune a enregistré : 1) une avancée du rivage dans quelques tronçons des trottoirs de la partie SE, 2) une progradation des embouchures de Oued Boussardoune, du canal d'irrigation provenant de Oued El Maouadine et de Oued Afelioun et 3) l'assèchement du marais salant de Kariat Arekman et de la bordure nord du Mont d'Atalayoun.

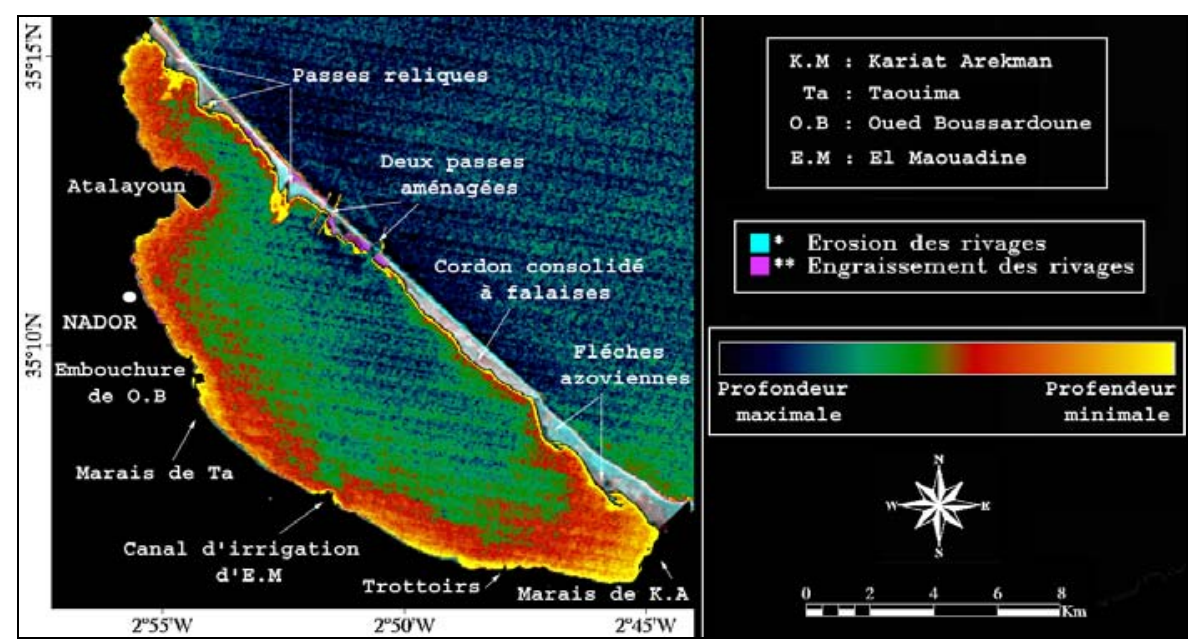

Fig. 2. Image de détection des changements du complexe lagunaire entre 1976 et 2010

\section{Conclusions}

Le suivi de l'évolution morphodynamique durant la période 2005 - 2010 montre clairement l'impact des aménagements sur l'équilibre sédimentaire et la configuration du complexe lagunaire de Nador. Les déséquilibres enregistrés pourraient entraîner la disparition d'une partie de l'île barrière. Ce risque est d'autant plus grand que d'une part la région est tectoniquement active et d'autre part, le réchauffement climatique global et ses conséquences (perturbations climatiques et élévation du niveau de la mer) sont inéluctables. 
La connaissance de la Mer :

un vecteur du développement durable en Méditerranée

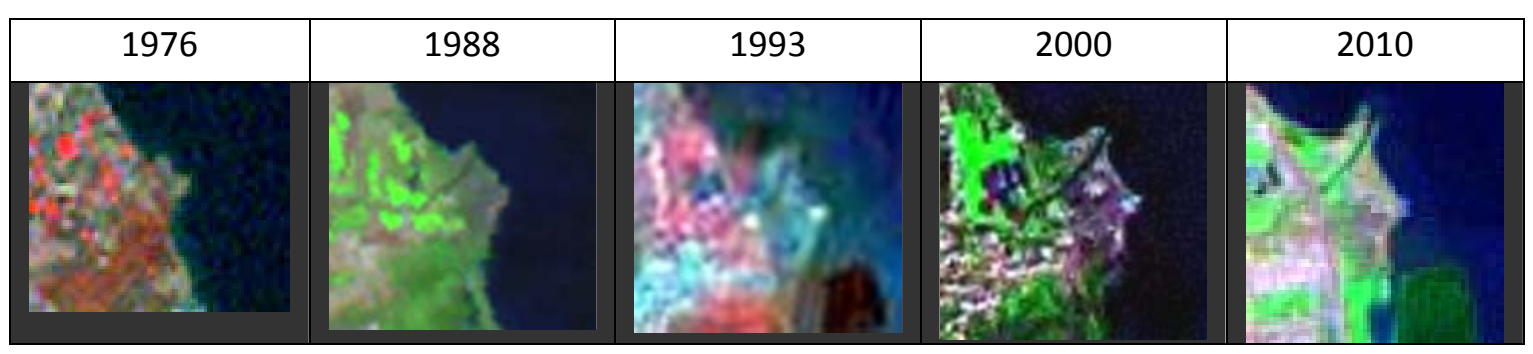

Fig. 3 : Evolution de l'embouchure d'Oued Bousardoune entre 1976 et 2010

\section{Références bibliographiques}

ERIMISCO P. (1961). La Mar Chica de Melilla, Bull. Int. Pêche Marit. Maroc, $\mathrm{n}^{\circ}$ 7, pp 3-11.

HAMOUMI N., IRZI Z. (1999). Les aménagements côtiers : problèmes et enseignements - Cas du littoral méditerranéen oriental, Actes de la $3^{\text {ème }}$ Rencontre hydrodynamique marine 99, $12 \mathrm{p}$.

HAMOUMI N., LOUAYA A (2005). Etude géomorphologique de la région de Nador, Rapport scientifique du Partenaire P3 (Maroc), Projet COLASU/ ICA3-2001-10029.

IRZI Z. (2002). Les environnements du littoral méditerranéen oriental du Maroc compris entre l'oued Kiss et le Cap des Trois Fourches; Dynamique sédimentaire et étude d'impact des sites aménagés et l'analyse des associations de foraminifères benthiques de la lagune de Nador en relation avec leur écologie. Thèse de Doctorat d’Etat. Université Mohammed Premier, Oujda, 279 p.

IRZI Z., HAMOUMI N. (1997). Les environnements sédimentaires du littoral méditerranéen oriental entre Saidia et le Cap des Trois Fourches, Maroc, Actes du 6 ème Congrès Français de Sédimentologie, Montpellier, Publ. ASF, Paris, n² 27, pp 151.

IRZI Z., KADIRI H., HAMOUMI N. (1997). Hydrologie et morphodynamique de l'île

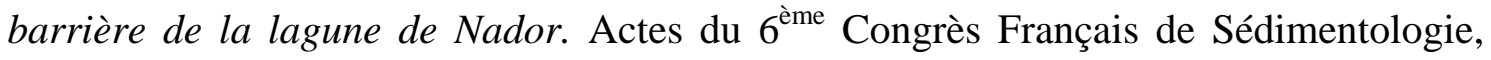
Montpellier, Publ. ASF, Paris, ${ }^{\circ} 27$, pp 149 -150.

LOUAYA A., HAMOUMI N. (2006). Application de la télédétection à l'étude de la géomorphologie et de la morphodynamique du complexe lagunaire de Nador. Bull. Geo Observateur, $\mathrm{n}^{\circ}$ 15, pp 51-65.

LOUAYA A., HAMOUMI N. (2010). Etude morphostructurale de la région de Nador (Maroc nord-oriental). Africa Géosciences Review, Vol. 17, № 2, pp 107-127

MAHJOUBI R. (1991). Évolution de la dynamique sédimentaire d'un système lagunaire microtidal pendant l'Holocène: lagune de Nador. Thèse de $3^{\text {ème }}$ cycle, Université. Med. Ben Abdellah, Fes, 289 p.

TESSON M. (1977). Etude hydrologique et hydrodynamique de la Sebkha Bou Areg (lagune de Nador, Maroc): bilan du printemps 1976. Trav. Doc. Inst. Pêches Marit., Casablanca, $\mathrm{n}^{\circ} 21$, pp 1-68. 